\title{
Collective excitations in quantum Hall liquid crystals: Single-mode approximation calculations
}

\author{
Cintia M. Lapilli and Carlos Wexler \\ Department of Physics and Astronomy, University of Missouri-Columbia, Columbia, Missouri 65211, USA \\ (Received 19 August 2005; revised manuscript received 30 December 2005; published 15 February 2006)
}

\begin{abstract}
A variety of recent experiments probing the low-temperature transport properties of quantum Hall systems have suggested an interpretation in terms of liquid crystalline mesophases dubbed quantum Hall liquid crystals. The single mode approximation (SMA) has been a useful tool for the determination of the excitation spectra of various systems such as phonons in ${ }^{4} \mathrm{He}$ and in the fractional quantum Hall effect. In this paper we calculate (via the SMA) the spectrum of collective excitations in a quantum Hall liquid crystal by considering nematic, tetratic, and hexatic generalizations of Laughlin's trial wave function having twofold, fourfold, and sixfold broken rotational symmetry, respectively. In the limit of zero wave vector $\mathbf{q}$ the dispersion of these modes is singular, with a gap that is dependent on the direction along which $\mathbf{q}=0$ is approached for nematic and tetratic liquid crystalline states, but remains regular in the hexatic state, as permitted by the fourth order wave-vector dependence of the (projected) oscillator strength and static structure factor.
\end{abstract}

DOI: 10.1103/PhysRevB.73.085314 PACS number(s): 73.43.-f, 52.27.Aj, 64.70.Md, 73.20.Mf

\section{INTRODUCTION}

For more than two decades two-dimensional electron systems (2DES), and in particular the quantum Hall effect $(\mathrm{QHE})^{1-3}$ have been constant sources of complex and unexpected behavior, perhaps with no equal in the realms of condensed matter physics. The unique combination of extremely high mobilities $\left(\mu \sim 10^{7} \mathrm{~m} / \mathrm{V} \mathrm{s}\right)$ in $\mathrm{GaAs} / \mathrm{Al}_{x} \mathrm{Ga}_{1-x}$ As heterostructures, low temperatures $(T<100 \mathrm{mK})$, enhancement of interactions due to the reduced dimensionality, and relative quenching of the kinetic energy in strong magnetic fields due to Landau level (LL) quantization, has allowed the emergence of complex and striking behavior due to subtle correlation effects. In fact, from the discovery of the integer and fractional QHE's in the early 1980s (Ref. 1) (leading to the award of two Nobel prizes), to the existence of novel fractionally charged ${ }^{2}$ and composite particles ${ }^{4}$ and many newer interesting many-body phenomena, the QHE has been a consistently active and exciting area of research.

The physics of 2DES in partially filled LL's is inherently complex due to the high degeneracy of the "unperturbed" ground state (i.e., without the Coulomb interaction). The first successful theoretical approach to this system was proposed shortly after the discovery of the $\mathrm{FQHE}^{1}$ by Laughlin, who proposed his famous trial wave function ${ }^{2}$

$$
\Psi_{1 / m}=\prod_{i<j}^{N}\left(z_{i}-z_{j}\right)^{m} e^{-\frac{1}{4} \sum_{k=1}^{N}\left|z_{k}\right|^{2}}
$$

to describe states at filling factor $\nu=1 / m$, with $m$ an odd integer. Here $z_{j}=x_{j}+i y_{j}$ is the position of the $j$ th electron in the complex plane and we work in units of the magnetic length $l_{0}=(\hbar / e B)^{1 / 2}$. The "goodness" of Laughlin state originates in that the nodal hypersurfaces on which the manybody wave function vanishes coincide with the ones in which the particles are in contact. Whereas the vanishing of the wave function when two (spin aligned) electrons are in contact is required by Fermi statistics, Laughlin state has mul- tiple nodes at those points, thus reducing the Coulomb repulsion.

Later, Jain proposed ${ }^{4}$ the elegant conceptual framework of the composite fermions $(\mathrm{CF})$ which unifies all hierarchies of integer and fractional QHE's, along with the intermediate states in between QH plateaus: an even number $2 p$ of vortices is attached to each electron, also lowering the Coulomb energy, and (in a "mean field" approximation) the "magnetic fluxes" associated with the vortices lead to a reduction of the effective magnetic field: $B^{*}=B-2 p \Phi_{0} n_{e}\left(\Phi_{0}=h / e\right.$ is the flux quantum and $n_{e}$ is the electron density), resulting in an effective filling factor $\nu^{*}=\nu /(1-2 p \nu)$. It is easy to see that for the strongest FQHE states $\nu^{*}$ is an integer (e.g., for $\nu=1 / 3$ and $p=1 \nu^{*}=1$ ), leading to the interpretation of the FQHE of electrons as a simple IQHE of the CF's; whereas the intermediate regions (e.g., $\nu=1 / 2,1 / 4$ ) correspond to $\nu^{*} \rightarrow \infty$, i.e., a vanishing effective magnetic field, $B^{*}=0$.

Since 1999, magnetotransport experiments have uncovered a variety of surprising results at low temperatures ( $T$ $\lesssim 100 \mathrm{mK}$ ), for example, extreme anisotropies ${ }^{5}$ and apparent competition between different ordered phases ${ }^{6}$ in the intermediate regions between quantum Hall plateaus at high LL's, the melting transition between a Wigner crystal (WC) at $\nu$ $\simeq 1 / 7,7$ and the microwave conductivity evidence of structural phase transitions in partially filled LL's. ${ }^{8}$ A large body of evidence corresponding to seemingly distinct phenomena may be partly understood in terms of a simple conceptual point: that the many-body states involved have an intrinsic crystalline or liquid crystalline order, ${ }^{9-17}$ be it smectic,,${ }^{9,11}$ nematic, ${ }^{10-13}$ tetratic or hexatic. ${ }^{13}$

Generalizations of Laughlin wave function [Eq. (1)] with discrete broken rotational symmetry (BRS) have been proposed in the past ${ }^{12,13,18}$ as candidates for nematic or hexatic states $^{12,13}$ in order to understand anisotropic transport observed in the intermediate regions, ${ }^{5}$ or the melting of the WC at $\nu=1 / 7 .^{7}$ In fact, the motivation for these states arises from the fact that it is generally expected that melting in 2D may occur through a topological Koszterlitz-Thouless-type (KT) transition. ${ }^{19}$ For 2D melting, the reliable Kosterlitz-Thouless- 


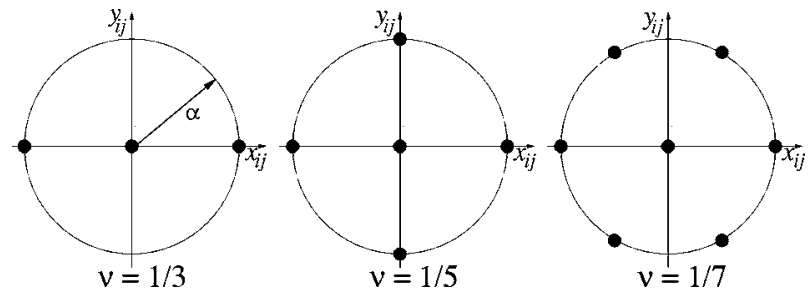

FIG. 1. Nodal distribution for $z_{i j} \equiv z_{i}-z_{j}$ for a quantum Hall nematic at $\nu=1 / 3$, tetratic at $\nu=1 / 5$, and hexatic at $\nu=1 / 7$.

Halperin-Nelson-Young (KTHNY) theory ${ }^{19,20}$ predicts that, in fact, an intermediate liquid crystalline phase will exist between a solid and a liquid phase, which will exhibit no translational order, and only a quasi-long-range order for the orientational order below the KT transition temperature. These arguments were used by Wexler and Dorsey ${ }^{11}$ to calculate qualitatively correct anisotropic-isotropic transition temperatures for the quantum Hall liquid crystal in the transitional regions at high LL's. ${ }^{5}$

In this paper we consider the spectrum of collective excitations for a family of liquid crystalline states in a partially filled LL. These states are generated so as to satisfy the following criteria which we consider reasonable for understanding the dynamics: (i) states must obey Fermi statistics, i.e., they must be odd under the exchange of any pair of electrons; (ii) the states must be translationally invariant (far enough from boundaries); (iii) there must be a broken rotational symmetry belonging to the proper symmetry group (i.e., $C_{2}$ for a nematic, $C_{4}$ for a tetratic, and $C_{6}$ for a hexatic; additional symmetries are possible in principle, e.g., with a $C_{10}$ symmetry, we have not explored such possibilities); (iv) states and excitations must reside entirely in the LLL to avoid the large cyclotron energy cost $\hbar \omega_{c}$. Note, as we will show later, that the properties of any excited LL may be readily obtained from the properties of the LLL.

States that satisfy the above-mentioned requirements have been proposed and studied in detail ${ }^{12,13,18}$ for filling factors $\nu=1 / 3,1 / 5$, and $1 / 7$. These are found by splitting the "extra" vortices of the Laughlin (or other CF) states around the electron, while obeying the required symmetries,

$$
\begin{aligned}
\Psi_{1 /(2 p+1)}^{\alpha}= & {\left[\prod_{i<j}^{N}\left(\prod_{\mu=1}^{2 p}\left(z_{i}-z_{j}-\alpha_{\mu}\right)\right)\right] } \\
& \times \prod_{i<j}^{N}\left(z_{i}-z_{j}\right) e^{-\frac{1}{4} \sum_{k=1}^{N}\left|z_{k}\right|^{2}},
\end{aligned}
$$

where the complex numbers $\alpha_{\mu}$ are distributed in pairs of opposite value in the complex plane (to satisfy Fermi statistics). For the states with the highest discrete symmetry at each filling factor we may take

$$
\alpha_{\mu}=\alpha e^{i 2 \pi(\mu-1) / 2 p}, \quad \mu \in\{1,2, \ldots, 2 p\},
$$

and without loss of generality $\alpha$ can be taken to be real. The wave function in Eq. (2) represents a homogeneous liquid crystalline state at $\nu=1 /(2 p+1)$, is antisymmetric, lies entirely in the LLL, and is smoothly connected to the isotropic Laughlin state for $\alpha=0$. Figure 1 depicts the nodal distribution for various states of Eq. (2). A remarkable feature of these states is that they possess an underlying charge density wave (CDW), but these CDWs are melted by fluctuations, and overall the system is translationally invariant. ${ }^{13}$

\section{THE SINGLE MODE APPROXIMATION}

To calculate the excitation spectrum we use the single mode approximation (SMA), ${ }^{21,22}$ which reliably provides the first moment (mean) of the energy of the excitations (for a given wave vector $\mathbf{k}$ ) that are coupled to the ground state by means of the density operator. ${ }^{21-23}$ The SMA was first used by Feynman in 1953 to accurately calculate the spectrum of phonons in superfluid ${ }^{4} \mathrm{He} .{ }^{21}$ The essence of the method originates on the assumption that the ground state of a system of bosons has a scarcity of long-wavelength excitations. Under those circumstances, the variational wave function for an excitation corresponding to a density wave can be written as

$$
\phi_{\mathbf{k}}\left(\mathbf{r}_{1}, \ldots, \mathbf{r}_{N}\right)=N^{-1 / 2} \rho_{\mathbf{k}} \psi_{0}\left(\mathbf{r}_{1}, \ldots, \mathbf{r}_{N}\right),
$$

where $\rho_{\mathbf{k}}=\sum_{j=1}^{N} e^{-i \mathbf{k} \cdot \mathbf{r}_{j}}$ is the density operator, and $\psi_{0}$ is the many-body ground state (which is, in fact, unknown for ${ }^{4} \mathrm{He}$ ). Note that this trial state automatically builds in the favorable correlations of the ground state. The energy of this excited state, $\Delta(\mathbf{k})=\left\langle\phi_{\mathbf{k}}\left|H-E_{0}\right| \phi_{\mathbf{k}}\right\rangle /\left\langle\phi_{\mathbf{k}} \mid \phi_{\mathbf{k}}\right\rangle$, can be simply evaluated,

$$
\Delta(\mathbf{k})=\frac{N^{-1}\left\langle\psi_{0}\left|\rho_{\mathbf{k}}^{\dagger}\left[H, \rho_{\mathbf{k}}\right]\right| \psi_{0}\right\rangle}{N^{-1}\left\langle\psi_{0}\left|\rho_{\mathbf{k}}^{\dagger} \rho_{\mathbf{k}}\right| \psi_{0}\right\rangle} \equiv \frac{f(\mathbf{k})}{S(\mathbf{k})} .
$$

In the last term the numerator is the "oscillator strength" and takes on the universal value $f(\mathbf{k})=\hbar^{2} k^{2} / 2 m$, and $S(\mathbf{k})$ is the static structure factor, which is directly measurable by means of neutron scattering (it is here, in fact, where the He-He correlations of the ground state are "encoded"). Using the experimental results for $S(\mathbf{k})$, Feynman could calculate a spectrum of remarkable quality, showing the phononlike spectrum at small wave vectors, and a roton minimum at

$$
v=1 / 3, \alpha=1.5 \quad v=1 / 5, \alpha=2.0 \quad v=1 / 7, \alpha=2.5
$$
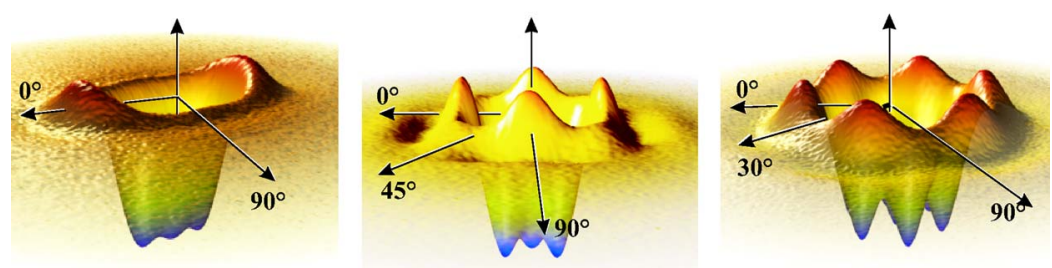

FIG. 2. (Color online) Pair correlation function $g(\mathbf{r})$ for a $\nu=1 / 3$ nematic, a $\nu=1 / 5$ tetratic, and a $\nu=1 / 7$ hexatic. In all cases $g(\mathbf{r})=O\left[r^{2 / \nu}\right]$ for small $r$ and $g(\infty) \rightarrow 1$. The fact that $g(\mathbf{r}) \simeq 1$ over a large area is a guarantee that the calculations are able to reproduce the thermodynamic limit. 

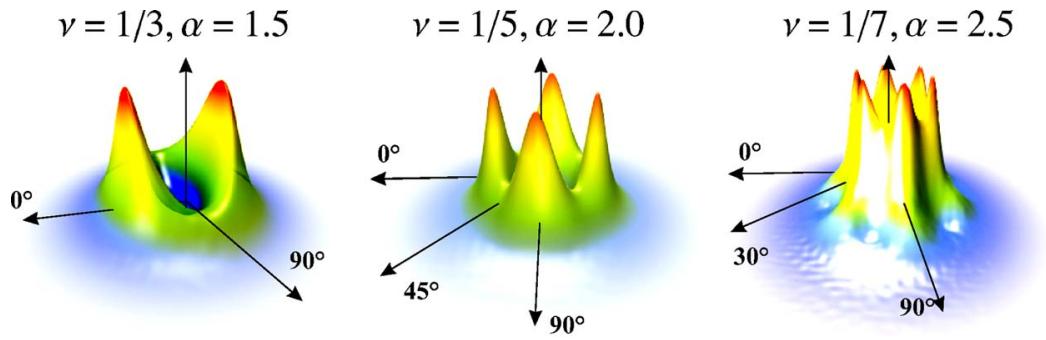

FIG. 3. (Color online) Projected static structure factors $\bar{S}(\mathbf{q})$ for a $\nu=1 / 3$ nematic, a $\nu=1 / 5$ tetratic, and a $\nu=1 / 7$ hexatic. In all cases, $\bar{S}(\mathbf{q})$ $=O\left(q^{4}\right)$ for small $q$, and $\bar{S}(\infty) \rightarrow 0$.

wave vectors comparable to the inverse interatomic distance. $^{21}$

\section{THE SINGLE MODE APPROXIMATION IN THE QUANTUM HALL EFFECT}

The applicability of the SMA to fermion systems is also well established for two- and three-dimensional systems in the absence of magnetic fields, giving a good approximation for the plasmons at long wavelength. For $2 \mathrm{DES}$ in presence of a magnetic field it correctly gives the zero-wave-vector magnetoplasmon at $\omega_{c}=e B / m_{e}$, a result that is guaranteed by Kohn's theorem, ${ }^{24}$ which states that the dipolar excitation is saturated by the cyclotron mode (this results in the modes of interest-the intra-LL excitations-having quadrupolar character, i.e., with an oscillator strength $\bar{f} \propto q^{4}$ ).

For excitations fully contained within a single LL, the cyclotron mode is not of primary interest. In 1985, Girvin, MacDonald, an Platzman (GMP) proposed an ingenious ansatz for projected excited states, ${ }^{22}$

$$
\left|\psi_{\mathbf{q}}\right\rangle=\bar{\rho}_{\mathbf{q}}\left|\psi_{0}\right\rangle
$$

where $\bar{\rho}_{\mathbf{q}}$ is the projected density operator ${ }^{25}$

$$
\begin{aligned}
\bar{\rho}_{\mathbf{q}} & =\sum_{m, m^{\prime}}\left\langle 0, m^{\prime}\left|e^{-i \mathbf{q} \cdot \mathbf{r}}\right| 0, m\right\rangle a_{0, m^{\prime}}^{\dagger} a_{0, m}=\sum_{j=1}^{N} \overline{e^{-i \mathbf{q} \cdot \mathbf{r}}} \\
& =\sum_{j=1}^{N} e^{-|q|^{2} / 2} e^{-i q^{*} z_{j} / 2} e^{-i q\left(\partial / \partial z_{j}\right)},
\end{aligned}
$$

where $|0, m\rangle$ correspond to single-particle states in the lowest LL and angular momentum $m$, and $a_{0, m}^{\dagger}$ is the particle creator operator for such state. As in Feynman's ansatz [Eq. (4)], Eq. (6) preserves the favorable correlations of the ground state. The exclusion of inter-LL excitations eliminates the problem with the saturation of the dipolar mode. The excited states have a compelling description, in first quantized form ${ }^{25}$

$$
\begin{aligned}
\bar{\rho}_{\mathbf{q}} \psi\left(z_{1}, \ldots, z_{N}\right)= & \sum_{j=1}^{N} e^{-|q|^{2} / 2} e^{-i q^{*} z_{j} / 2} \\
& \times \psi\left(z_{1}, \ldots, z_{j-1}, z_{j}-i q, z_{j+1}, \ldots, z_{N}\right),
\end{aligned}
$$

which corresponds to shifting each electron by $\hat{e}_{z} \times \mathbf{q}$ and superimposing these $N$ configurations with an amplitude $e^{-i q^{*} z_{j} / 2}$.

Similarly to Eq. (5), the excitation spectrum can be readily obtained

$$
\bar{\Delta}_{\mathbf{q}}=\frac{(2 N)^{-1}\left\langle\psi_{0}\left|\left[\bar{\rho}_{\mathbf{q}}^{\dagger},\left[\bar{H}, \bar{\rho}_{\mathbf{q}}\right]\right]\right| \psi_{0}\right\rangle}{N^{-1}\left\langle\psi_{0}\left|\bar{\rho}_{\mathbf{q}}^{\dagger} \bar{\rho}_{\mathbf{q}}\right| \psi_{0}\right\rangle} \equiv \frac{\bar{f}(\mathbf{q})}{\bar{S}(\mathbf{q})} .
$$

The projected oscillator strength comes from the noncommutation of the projected density operator with terms in the potential energy part of the Hamiltonian also projected onto the LLL,

$$
\bar{H}=\frac{1}{2} \sum_{\mathbf{q}} v_{\mathbf{q}}\left(\bar{\rho}_{\mathbf{q}}^{\dagger} \bar{\rho}_{\mathbf{q}}-N e^{-q^{2} l^{2} / 2}\right)
$$

Since $\left[\bar{\rho}_{\mathbf{k}}, \bar{\rho}_{\mathbf{q}}\right]=\left(e^{k^{*} q / 2}-e^{k q^{*} / 2}\right) \bar{\rho}_{\mathbf{k}+\mathbf{q}}$, we find ${ }^{22}$

$$
\bar{f}(\mathbf{q})=2 e^{-|q|^{2} / 2} \sum_{\mathbf{k}} \sin ^{2}\left(\frac{\mathbf{q} \times \mathbf{k}}{2}\right) \bar{S}(\mathbf{k})\left(v_{\mathbf{k}-\mathbf{q}} e^{\mathbf{k} \cdot \mathbf{q}} e^{-|q|^{2} / 2}-v_{\mathbf{k}}\right)
$$

For its part, the projected static structure factor $\bar{S}(\mathbf{q})$ can be calculated from ${ }^{22,25}$

$$
\bar{S}(\mathbf{q})=S(\mathbf{q})-\left(1-e^{-q^{2} / 2}\right),
$$

where $S(\mathbf{q})$ is the unprojected static structure factor,
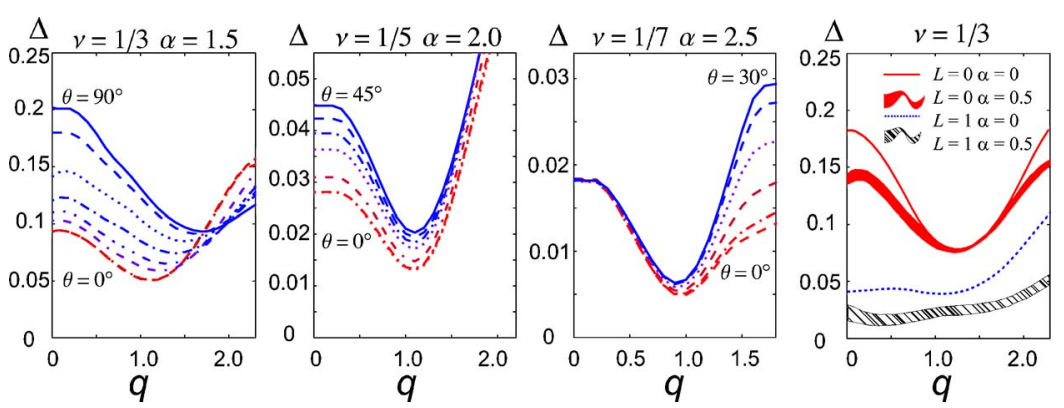

FIG. 4. (Color online) Single mode approximation spectra for a $\nu=1 / 3$ nematic, a $\nu=1 / 5$ tetratic, and a $\nu=1 / 7$ hexatic. Note the dramatic angular dependence of the spectra, and the appearance of a singular gap as $q \rightarrow 0$ for the nematic and tetratic. The rightmost panel shows also the spectum for the $\nu=1 / 3$ nematic in the first excited LL $(L=1)$. 


$$
S(\mathbf{q})-1=\rho_{0} \int d^{2} r e^{-i \mathbf{q} \cdot \mathbf{r}}[g(\mathbf{r})-1],
$$

the Fourier transform of the pair correlation function

$$
g(\mathbf{r})=n_{e}^{-2}\left\langle\sum_{i \neq j}^{N} \delta\left(\mathbf{r}_{i}-\mathbf{r}\right) \delta\left(\mathbf{r}_{j}\right)\right\rangle
$$

which is obtainable from the ground state via, e.g., Monte Carlo (MC) simulations. ${ }^{12,13,22}$ In our case, we considered BRS states [Eq. (2)] corresponding to a $\nu=1 / 3$ nematic, a $\nu=1 / 5$ tetratic, and a $\nu=1 / 7$ hexatic. Figure 2 depicts the pair correlation function for various states. In all cases, correlation functions and SMA excitation spectra were computed for numerous $\alpha$ 's. The angle dependence significantly increases the burden in the MC simulations since the full angle dependent $g(\mathbf{r})$ is needed, rather than the considerably simpler angle averaged $g(r)$ of the isotropic systems. The accurate calculation of $\bar{f}(\mathbf{q})$, with its angular-dependent exponentially large factors required high quality $\bar{S}(\mathbf{q})$ and hence $g(\mathbf{r})$. To put things in perspective, to achieve ca. $1 \%$ accuracy in $g(\mathbf{r}), O[10,000]$ counts were accumulated for each $0.01 \times 0.01$ (in units of $l_{0}$ ) $\mathbf{r}$-box in the original histograms (the small boxes were necessary to have precision in the Fourier transform at high wave vectors). This required, for each filling factor $\nu$ and anisotropy generating $\alpha$, to run approximately $4-8 \times 10^{7} \mathrm{MC}$ steps in systems of $N_{e}$ $=200-400$ electrons taking 100-200 cpu $\times$ days of computation for each $(\nu, \alpha)$ in a $2 \mathrm{GHz}$ Athlon cluster (see Ref. 13 for further details). A relatively large $N_{e}$ is required so that the simulations are able to reproduce a system in the thermodynamic limit. The fact that $g(\mathbf{r}) \simeq 1$ over a large area is a guarantee of that achievement (Fig. 2).

Figure 3 shows the projected static structure factors $\bar{S}(\mathbf{q})$ for a $\nu=1 / 3$ nematic, a $\nu=1 / 5$ tetratic, and a $\nu=1 / 7$ hexatic. From $\bar{S}(\mathbf{q})$, the oscillator strength $\bar{f}(\mathbf{q})$ is computed using Eq. (11). Analysis of $\bar{S}(\mathbf{q})$ and $\bar{f}(\mathbf{q})$ shows that both are $O\left[q^{4}\right]$ for small q. ${ }^{22}$ This restriction on the small wavevector behavior originates in Kohn's theorem, ${ }^{24}$ as all of the $O\left(q^{2}\right)$ pieces in the unprojected parts are saturated by the (uninteresting) inter-LL excitations at $\hbar \omega_{c}$.

Figure 4 presents some of our results for the excitation spectra in the lowest LL. The results are consistent with those obtained by GMP for the isotropic $\nu=1 / 3$ and $1 / 5$ FQHE cases $^{22}$ which were qualitatively confirmed experimentally. ${ }^{26}$ They show that the collective excitation spectrum remains gapped, albeit with a deep magnetoroton, for modes coupled to the ground state via the density operator. Not surprisingly, BRS states have significant anisotropy in their spectra. However, it is noteworthy that for the nematic and tetratic cases the spectrum is singular, with an angle dependence on the excitation energy $\Delta(\mathbf{q})$ as $\mathbf{q} \rightarrow 0$. By contrast, the hexatic liquid crystal has a regular spectrum in the long wavelength limit. The apparent disparity have, of course, to do with the different rotational symmetries of the different states: as $\bar{\Delta}=\bar{f} / \bar{S}$, and both numerator and denominator are $O\left(q^{4}\right)$ for small $\mathbf{q}$, there is no possibility of gener-

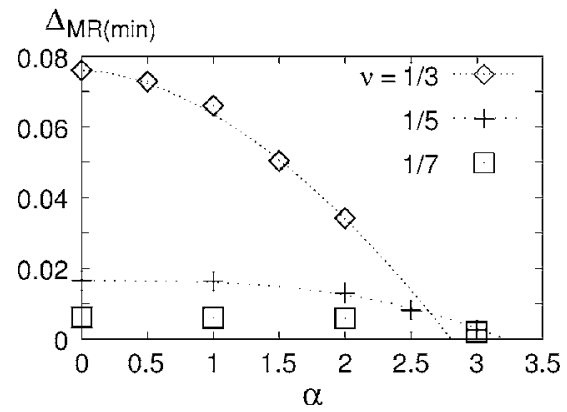

FIG. 5. Possible collapse of the magnetoroton minimum as function of the anisotropy parameter $\alpha$. Dotted lines are guides to the eye.

ating a $C_{6}$ symmetric form with terms that can only depend on $q_{x}^{4}, q_{y}^{4}$, and $q_{x}^{2} q_{y}^{2}$. This can also be understood from the point of view of an effective elasticity theory for 2DES (valid in the long wavelength limit, $q \rightarrow 0$ ): ${ }^{27}$ the elasticity tensor being of the fourth rank is not compatible with a sixfold rotational symmetry. This elastic interpretation will be presented elsewhere. ${ }^{17}$

The presence of this singular spectrum suggests that microwave conductivity experiments ${ }^{8,26}$ may be able to discern such structures as a signature of, e.g., the quantum Hall nematic suggested by magnetotransport experiments. ${ }^{5,6}$

Another interesting feature (Fig. 5) is that the magnetoroton minima and the gap at the origin appears to collapse at some finite anisotropy factor $\alpha$. The appearance of elastic modes that go soft may be a precursor of the appearance of charge density waves in the system. ${ }^{13}$ Spectra in higher LL's can be obtained by multiplying the projected density operator $\bar{\rho}_{\mathbf{q}}$ by $L_{L}\left(q^{2} / 2\right)$, where $L_{L}(x)$ is the Laguerre polynomial and $L$ corresponds to the desired LL. ${ }^{28}$ The rightmost panel of Fig. 4 shows the modification of the SMA spectrum for the first excited LL. Numerical error due to the large $L_{L}\left(q^{2} / 2\right)$ factors makes it difficult to get reliable results for higher LL's at this point.

Theoretical predictions of which type of order is lowest in free energy (required to decide which state is favorable at finite temperatures, as shown in Refs. 6 and 7) are still incomplete, however, as the entropy is likely to be dominated by gapless modes originating, e.g., from the Goldstone modes associated with the spontaneous breaking of the continuous rotational symmetry of the isotropic states. Generalization of the SMA to gapless rotational modes (see, e.g., Ref. 15) will require, however, three-body operators which demanding considerably higher computing capabilities. ${ }^{29}$

\section{SUMMARY}

Summarizing, in connection with the recent variety of experimental evidence supporting liquid crystalline phases in quantum Hall systems, ${ }^{5-8}$ we have calculated the collective excitation spectrum in the SMA approximation for a quantum Hall liquid crystal. We found that the spectrum of excitations coupled to the ground state by the density operator remains gapped, but develops a significant anisotropy, which in the case of the nematic and tetratic liquid crystals has a singular gap in the long wavelength limit. 


\section{ACKNOWLEDGMENTS}

The authors would like to acknowledge helpful discussions with G. Vignale, O. Ciftja, A.T. Dorsey, M. Fogler, A. MacDonald, S. Girvin, E. Fradkin, H. Fertig, and J. Jain.
Acknowledgment is made to the University of Missouri Research Board and Research Council, and to the Donors of the Petroleum Research Fund, administered by the American Chemical Society, for support of this research.
${ }^{1}$ K. v. Klitzing, G. Dorda, and M. Pepper, Phys. Rev. Lett. 45, 494 (1980); D. C. Tsui, H. L. Stormer, and A. C. Gossard, ibid. 48, 1559 (1982).

${ }^{2}$ R. B. Laughlin, Phys. Rev. Lett. 50, 1395 (1983).

${ }^{3}$ For reviews see Perspectives in Quantum Hall Effects, edited by S. Das Sarma and A. Pinczuk (Wiley, New York, 1996); The Quantum Hall Effect, 2nd ed., edited by R. E. Prange and S. M. Girvin (Springer-Verlag, New York, 1990).

${ }^{4}$ J. Jain, Phys. Today 53, 39 (2000); in Composite Fermions, edited by O. Heinonen (World Scientific, New York, 1998).

${ }^{5}$ M. P. Lilly, K. B. Cooper, J. P. Eisenstein, L. N. Pfeiffer, and K. W. West, Phys. Rev. Lett. 82, 394 (1999); R. R. Du, D. C. Tsui, H. L. Stormer, L. N. Pfeiffer, K. W. Baldwin, and K. W. West, Solid State Commun. 109, 389 (1999).

${ }^{6}$ G. Gervais, L. W. Engel, H. L. Stormer, D. C. Tsui, K. W. Baldwin, K. W. West, and L. N. Pfeiffer, Phys. Rev. Lett. 93, 266804 (2004).

${ }^{7}$ W. Pan, H. L. Stormer, D. C. Tsui, L. N. Pfeiffer, K. W. Baldwin, and K. W. West, Phys. Rev. Lett. 88, 176802 (2002).

${ }^{8}$ R. M. Lewis, Y. Chen, L. W. Engel, D. C. Tsui, P. D. Ye, L. N. Pfeiffer, and K. W. West Phys. Rev. Lett. 93, 176808 (2004).

${ }^{9}$ M. M. Fogler, A. A. Koulakov, and B. I. Shklovskii, Phys. Rev. B 54, 1853 (1996); R. Moessner and J. T. Chalker, ibid. 54, 5006 (1996).

${ }^{10}$ E. Fradkin and S. A. Kivelson, Phys. Rev. B 59, 8065 (1999); S. A. Kivelson, E. Fradkin, and V. J. Emery, Nature (London) 393, 550 (1998).

${ }^{11}$ C. Wexler and A. T. Dorsey, Phys. Rev. B 64, 115312 (2001).

${ }^{12}$ O. Ciftja and C. Wexler, Phys. Rev. B 65, 045306 (2002); 65, 205307 (2002); A. J. Schmidt, O. Ciftja, and C. Wexler, ibid. 67, 155315 (2003); C. Wexler and O. Ciftja, J. Phys.: Condens. Matter 14, 3705 (2002).

${ }^{13}$ O. Ciftja, C. M. Lapilli, and C. Wexler, Phys. Rev. B 69, 125320
(2004).

${ }^{14}$ A. H. MacDonald and M. P. A. Fisher, Phys. Rev. B 61, 5724 (2000); F. von Oppen, B. I. Halperin, and A. Stern, Phys. Rev. Lett. 84, 2937 (1999); S.-Y. Lee, V. W. Scarola, and J. K. Jain, ibid. 87, 256803 (2001).

${ }^{15}$ L. Radzihovsky and A. T. Dorsey, Phys. Rev. Lett. 88, 216802 (2002); M. M. Fogler, Europhys. Lett. 66, 572 (2004).

${ }^{16}$ C. M. Lapilli and C. Wexler, in ICPS27, edited by J. Menendez and C. van de Walle, AIP Conf. Proc. (AIP, New York, 2005); C. Wexler and C. M. Lapilli, in Condensed Matter Theories, edited by R. M. Panoff and J. W. Clark (Nova Science, New York, 2005), Vol. 20.

${ }^{17}$ C. M. Lapilli, C. Wexler, and G. Vignale, Bull. Am. Phys. Soc. 50, 1272 (2005).

${ }^{18}$ K. Musaelian and R. Joynt, J. Phys.: Condens. Matter 8, L105 (1996); L. Balents, Europhys. Lett. 33, 291 (1996).

${ }^{19}$ J. M. Kosterlitz and D. J. Thouless, J. Phys. C 6, 1181 (1973); J. M. Kosterlitz, ibid. 7, 1046 (1974).

${ }^{20}$ B. I. Halperin and D. R. Nelson, Phys. Rev. Lett. 41, 121 (1978); A. P. Young, Phys. Rev. B 19, 1855 (1979).

${ }^{21}$ R. P. Feynman, Phys. Rev. 91, 1291 (1953); 94, 262 (1954).

${ }^{22}$ S. M. Girvin, A. H. MacDonald, and P. M. Platzman, Phys. Rev. Lett. 54, 581 (1985); Phys. Rev. B 33, 2481 (1986).

${ }^{23}$ K. Park and J. K. Jain, Solid State Commun. 115, 353 (2000).

${ }^{24}$ W. Kohn, Phys. Rev. 123, 1242 (1961).

${ }^{25}$ S. M. Girvin, Phys. Rev. B 30, 558 (1984).

${ }^{26}$ A. Pinczuk, B. S. Dennis, L. N. Pfeiffer, and K. West, Phys. Rev. Lett. 70, 3983 (1993); Physica B 249, 40 (1998).

${ }^{27}$ S. Conti and G. Vignale, J. Phys.: Condens. Matter 10, L779 (1998).

${ }^{28}$ See e.g., A. H. MacDonald and S. M. Girvin, Phys. Rev. B 33, 4009 (1986); also see Refs. 12 and 13.

${ }^{29}$ M. M. Fogler (private communication). 